\title{
Universally Composable Secure Computation with (Malicious) Physically Uncloneable Functions
}

\author{
Rafail Ostrovsky $^{1,2}$, Alessandra Scafuro $^{1}$, Ivan Visconti $^{3}$, and Akshay Wadia ${ }^{1}$ \\ 1 Department of Computer Science, UCLA, USA \\ 2 Department of Mathematics, UCLA, USA \\ \{rafail, scafuro, awadia\}@cs.ucla.edu \\ 3 Dipartimento di Informatica, University of Salerno, Italy \\ visconti@dia.unisa.it
}

\begin{abstract}
Physically Uncloneable Functions (PUFs) 28] are noisy physical sources of randomness. As such, they are naturally appealing for cryptographic applications, and have caught the interest of both theoreticians and practitioners. A major step towards understanding and securely using PUFs was recently taken in [Crypto 2011] where Brzuska, Fischlin, Schröder and Katzenbeisser model PUFs in the Universal Composition (UC) framework of Canetti [FOCS 2001]. A salient feature of their model is that it considers trusted PUFs only; that is, PUFs which have been produced via the prescribed manufacturing process and are guaranteed to be free of any adversarial influence. However, this does not accurately reflect real-life scenarios, where an adversary could be able to create and use malicious PUFs.

The goal of this work is to extend the model proposed in [Crypto 2011] in order to capture such a real-world attack. The main contribution of this work is the study of the Malicious PUFs model. To this end, we first formalize the notion of "malicious" PUFs, and extend the UC formulation of Brzuska et al. to allow the adversary to create PUFs with arbitrary adversarial behaviour. Then, we provide positive results in this, more realistic, model. We show that, under computational assumptions, it is possible to UC-securely realize any functionality.
\end{abstract}

\section{Introduction}

The impossibility of secure computation in the universal composability framework was proved first by Canetti and Fischlin [9], and then strengthened by Canetti et al. in [10]. Impossibility of even weaker notions has been proved in $[1,5,16$.

As a consequence, several setup assumptions, and relaxations of the UC framework have been proposed to achieve UC security [4, 11, 19, 29].

In recent years, researchers have started exploring the use of secure hardware in protocol design. The idea is to achieve protocols with strong security guarantees (like UC) by allowing parties to use hardware boxes that have certain

T. Johansson and P. Nguyen (Eds.): EUROCRYPT 2013, LNCS 7881, pp. 702-718, 2013.

(C) International Association for Cryptologic Research 2013 
security properties. An example of the kind of security required from such a hardware box is that of tamper-proofness; i.e., the receiver of the box can only observe the input/output behaviour of the functionality that the box implements. This property was formalized by Katz in 20, and it was shown that UC security is possible by relying on the existence of tamper-proof programmable hardware tokens, and computational assumptions. Smart cards are well understood examples of such tokens, since they have been used in practice in the last decades. Several improvements and variations of Katz's model have been then proposed in follow up papers (e.g., [17]).

Spurred by technological advances in manufacturing, recently a new hardware component has gained a lot of attention: Physically Uncloneable Functions (PUFs) 27]28. A PUF is a hardware device generated through a special physical process that implements a "random" function 1 that depends upon the physical parameters of the process. These parameters can not be "controlled", and producing a clone of the device is considered infeasible 2 . Once a PUF has been constructed, there is a physical procedure to query it, and to measure its answers. The answer of a PUF depends on the physical behavior of the PUF itself, and is assumed to be unpredictable, or to have high min-entropy. Namely, even after obtaining many challenge-response pairs, it is infeasible to predict the response to a new challenge.

Since their introduction by Pappu in 2001, PUFs have gained a lot of attention for cryptographic applications like anti-counterfeiting mechanisms, secure storage, RFID applications, identification and authentication protocols [14, 15, $18,18,22,33,34$. More recently PUFs have been used for designing more advanced cryptographic primitives. In 31] Rührmair shows the first construction of Oblivious Transfer, the security proof of which is later provided in [32]. In [3], Armknecht et al. deploy PUFs for the construction of memory leakage-resilient encryption schemes. In [23] Maes et al. provide construction and implementation of PUFKY, a design for PUF-based cryptographic key generators. There exist several implementations of PUFs, often exhibiting different properties. The work of Armknecht et al. 22 formalizes the security features of physical functions in accordance to existing literature on PUFs and proposes a general security framework for physical functions. A survey on PUF implementations is given in 24]. Very recently in 21. Katzenbeisser et al. presented the first large scale evaluation of the security properties of some popular PUFs implementations (i.e., intrinsic electronic PUFs).

Modeling PUFs in the UC Framework. Only very recently, Brzuska et al. 7] suggested a model for using PUFs in the UC setting that aims at abstracting real-world implementations. The unpredictability and uncloneability properties are modeled through an ideal functionality. Such functionality allows only the

\footnotetext{
1 Technically, a PUF does not implement a function in the mathematical sense, as the same input might produce different responses.

${ }^{2}$ SRAM PUFs (memory-based PUFs) might be cloneable according to recent finding 6 .
} 
creation of trusted PUFs. In 7] PUFs are thought as non-PPT setup assumptions. As such, a PPT simulator cannot simulate a PUF, that is, PUFs are nonprogrammable. Although non-programmable, PUFs are not modeled as global setup [8]. 7] shows how to achieve unconditional UC secure Oblivious Transfer, Bit Commitment and Key Agreement with trusted PUFs.

\subsection{Our Contribution}

We observe that the UC formulation of PUFs proposed by Brzuska et al. makes the following crucial assumption: the model considers trusted PUFs only, that is, adversaries are assumed to be unable to produce fake/malicious PUFs. As we argue below, we feel that assuming that an adversary cannot misbehave by creating fake/malicious PUFs, might be unrealistic in the real world. Given that the study of PUFs is still in its infancy, it is risky to rely on assumptions on the impossibility of the adversaries in generating and accessing PUFs adversarially. The main contribution of this work is to study security models that capture such plausible real-world attacks, and provide protocols that are secure in the presence of such adversaries.

Modeling malicious PUFs. We augment the UC framework so to enable the adversary to create untrusted (malicious) PUFs. But what exactly are malicious PUFs? In real life, an adversary could tamper with a PUF in such a way that the PUF loses any of its security properties. Or the adversary may introduce new behaviours; for example, the PUF may start logging its queries. To keep the treatment of malicious behaviour as general as possible, we allow the adversary to send as PUF any hardware token that meets the syntactical requirements of a PUF. Thus, an adversary is assumed to be able to even produce fake PUFs that might be stateful and programmed with malicious code. We assume that a malicious PUF however cannot interact with its creator once is sent away to another party. If this was not the case, then we are back in the standard model (see the Introduction in the full version [26]).

UC secure computation with malicious PUFs. The natural question is whether UC security can be achieved in such a much more hostile setting. We give a positive answer to this question by constructing a computational UC commitment scheme in the malicious PUFs model. Our commitment scheme needs two PUFs that are transferred only once (PUFs do not go back-and-forth), at the beginning of the protocol and it requires computational assumptions. We avoid that PUFs go back-and-forth by employing a technique that requires OT. The results of Canetti, et al. [1] shows how to achieve general UC computation from computational UC commitments. Whether unconditional UC secure computation is possible in the malicious PUF model, is still an open problem.

Hardness assumptions with PUFs. Notice that as correctly observed in [7], since PUFs are not PPT machines, it is not clear if standard complexity-theoretic 
assumptions still hold in presence of PUFs. We agree with this observation. However the critical point is that even though there can exist a PUF that helps to break in polynomial time a standard complexity-theoretic assumptions, it is still unlikely that a PPT adversary can find such a PUF. Indeed a PPT machine can only generate a polynomial number of PUFs, therefore obtaining the one that allows to break complexity assumptions is an event that happens with negligible probability and thus it does not effect the concrete security of the protocols.

In light of the above discussion, only one of the following two cases is possible. 1) Standard complexity-theoretic assumptions still hold in presence of PPT adversaries that generate PUFs; in this case our construction is secure. 2) There exists a PPT adversary that can generate a PUF that breaks standard assumptions; in this case our construction is not secure, but the whole foundations of complexity-theoretic cryptography would fall down (which is quite unlikely to happen) with respect to real-world adversaries. We elaborate on this issue in Section 3.1

Additional results. We now mention additional results that can be found in the full version of this paper [26] but have been omitted from the present conference version due to lack of space. Firstly, we further investigate the feasibility of achieving unconditional security in the malicious PUF model. We leave the important question of unconditional UC open, but provide a construction of an unconditional commitment scheme in the malicious PUF model. Secondly, we propose and study another modification to the original model of Brzuska et al. In the new model which we call "oblivious-query model", all parties (and the adversary) use trusted PUFs, but in the security proofs, the simulator is not allowed to observe the adversary's queries to its PUF. The main motivation for studying this modification is that the ability of the simulator to observe adversary's queries stems from the assumption that there is only a single, prescribed procedure for evaluating a PUF. As we discuss in detail in the full version, this assumption is not well-justified in the real world. Our main contribution in the oblivious-query model is the construction of an unconditional UC protocol for OT. Lastly, we show that if both adversarial modes discussed above are combined, viz., adversaries can create malicious PUFs and may query honest PUFs via non-prescribed processes, then UC security is impossible.

Independent work. Very recently and independently of us, van Dijk and Rührmair 36] also study the use of PUFs in cryptographic protocols. Among other things, they consider the "bad" PUF model where PUFs can be augmented with malicious behaviour like keeping a log of queries, etc. They show that unconditional OT is impossible using bad PUFs, but their setting is very different from ours. For a detailed discussion about the work of van Dijk and Rührmair, see the Introduction of the full version [26]. 


\section{Definitions}

Notation. We let $n$ be the security parameter and PPT be the class of probabilistic polynomial time Turing machines. We use $v \stackrel{\$}{\leftarrow} A()$ when the algorithm $A$ is randomized. We denote by disham $(a, b)$ the Hamming distance of $a$ and $b$.

Physically uncloneable functions. We follow definitions given in [7]. A PUF is a noisy physical source of randomness. The randomness property comes from an uncontrollable manufacturing process. A PUF is evaluated with a physical stimulus, called the challenge, and its physical output, called the response, is measured. Because the processes involved are physical, the function implemented by a PUF can not (necessarily) be modeled as a mathematical function, neither can be considered computable in PPT. Moreover, the output of a PUF is noisy, namely, querying a PUF twice with the same challenge, could yield to different outputs. The mathematical formalization of a PUF due to [7] is the following.

A PUF-family $\mathcal{P}$ is a pair of (not necessarily efficient) algorithms Sample and Eval, and is parameterized by the bound on the noise of PUF's response $d_{\text {noise }}$ and the range of the PUF's output $r g$. Algorithm Sample abstracts the PUF fabrication process and works as follows. On input the security parameter, it outputs a PUF-index id from the PUF-family satisfying the security property (that we define soon) according to the security parameter. Algorithm Eval abstracts the PUF-evaluation process. On input a challenge $q$, it evaluates the PUF on $q$ and outputs the response $a$ of length $r g$. The output is guaranteed to have bounded noise $d_{\text {noise }}$, meaning that, when running $\operatorname{Eval}\left(1^{n}\right.$, id, $\left.q\right)$ twice, the Hamming distance of any two responses $a_{1}, a_{2}$ is smaller than $d_{\text {noise }}(n)$. Wlog, we assume that the challenge space of a PUF is a full set of strings of a certain length.

Definition 1 (Physically Uncloneable Functions). Let rg denote the size of the range of the PUF responses of a PUF-family and $d_{\text {noise }}$ denote a bound of the PUF's noise. $\mathcal{P}=\left(\right.$ Sample, Eval) is a family of $\left(r g, d_{\text {noise }}\right)-P U F$ if it satisfies the following properties.

Index Sampling. Let $\mathcal{I}_{n}$ be an index set. On input the security parameter $n$, the sampling algorithm Sample outputs an index id $\in \mathcal{I}_{n}$ following a not necessarily efficient procedure. Each id $\in \mathcal{I}_{n}$ corresponds to a set of distributions $\mathcal{D}_{\mathrm{id}}$. For each challenge $q \in\{0,1\}^{n}, \mathcal{D}_{\text {id }}$ contains a distribution $\mathcal{D}_{\text {id }}(q)$ on $\{0,1\}^{r g(n)} . \mathcal{D}_{\text {id }}$ is not necessarily an efficiently sampleable distribution.

Evaluation. On input the tuple $\left(1^{n}, \mathrm{id}, q\right)$, where $q \in\{0,1\}^{n}$, the evaluation algorithm Eval outputs a response $a \in\{0,1\}^{r g(n)}$ according to distribution $\mathcal{D}_{\text {id }}(q)$. It is not required that Eval is a PPT algorithm.

Bounded Noise. For all indexes id $\in \mathcal{I}_{n}$, for all challenges $q \in\{0,1\}^{n}$, when running $\mathrm{Eval}\left(1^{n}, \mathrm{id}, q\right)$ twice, the Hamming distance of any two responses $a_{1}, a_{2}$ is smaller than $d_{\text {noise }}(n)$.

In the paper we use $\operatorname{PUF}_{\text {id }}(q)$ to denote $\mathcal{D}_{\text {id }}(q)$. When not misleading, we omit id from PUF $_{\text {id }}$, using only the notation PUF. 
Security of PUFs. We assume that PUFs enjoy the properties of uncloneability and unpredictability. Unpredictability is modeled via an entropy condition on the PUF distribution. Namely, given that a PUF has been measured on a polynomial number of challenges, the response of the PUF evaluated on a new challenge has still a significant amount of entropy. Formally,

Definition 2 (Unpredictability). $A\left(r g, d_{\text {noise }}\right)-P U F$ family $\mathcal{P}=($ Sample, Eval) for security parameter $n$ is $\left(d_{\min }(n), m(n)\right)$-unpredictable if for any $q \in\{0,1\}^{n}$ and challenge list $\mathcal{Q}=\left(q_{1}, \ldots, q_{\mathrm{poly}(n)}\right)$, one has that, if for all $1 \leq k \leq$ poly $(n)$ the Hamming distance satisfies $\operatorname{disham}_{\text {ham }}\left(q, q_{k}\right) \geq d_{\min }(n)$, then the average min-entropy satisfies $\tilde{H}_{\infty}(\operatorname{PUF}(q) \mid \operatorname{PUF}(\mathcal{Q})) \geq m(n)$, where $\operatorname{PUF}(\mathcal{Q})$ denotes a sequence of random variables $\operatorname{PUF}\left(q_{1}\right), \ldots, \operatorname{PUF}\left(q_{\mathrm{poly}(n)}\right)$ each corresponding to an evaluation of the PUF on challenge $q_{k}$. Such a PUF-family is called a $\left(r g, d_{\text {noise }}, d_{\min }, m\right)-P U F$ family.

Fuzzy extractors. Fuzzy extractors of Dodis et al. [13] are applied to the outputs of the PUF, to convert such noisy, high-entropy measurements into reproducible randomness. Very informally, a fuzzy extractor is a pair of efficient randomized algorithms (FuzGen, FuzRep). FuzGen takes as input an $\ell$-bit string, that is the PUF's response $a$, and outputs a pair $(p, s t)$, where $s t$ is a uniformly distributed string, and $p$ is a public helper data string. FuzRep takes as input the PUF's noisy response $a^{\prime}$ and the helper data $p$ and generates the very same string $s t$ obtained with the original measurement $a$. The security property of fuzzy extractors guarantees that, if the min-entropy of the PUF's responses are greater than a certain parameter $m$, knowledge of the public data $p$ only, without the measurement $a$, does not give any information on the secret value $s t$. The correctness property, guarantees that, all pairs of responses $a, a^{\prime}$ that are close enough, i.e., their hamming distance is less then a certain parameter $t$, will be recovered by FuzRep to the same value st generated by FuzGen. In order to apply fuzzy extractors to PUF's answers, it is sufficient to pick an extractor whose parameters match with the parameter of the PUF being used.

\section{UC Security with Malicious PUFs}

In Section 1 we have motivated the need of a different formulation of UC security with PUFs that allows the adversary to generate malicious PUFs. In this section we first show how to model malicious PUFs in the UC framework, and then show that as long as standard computational assumptions still hold when PPT adversaries can generate (even malicious) PUFs, there exist protocols for UC realizing any functionality with (malicious) PUFs.

\subsection{Modeling Malicious PUFs}

We allow our adversaries to send malicious PUFs to honest parties? As discussed before, the motivation for malicious PUFs is that the adversary may have some

${ }^{3}$ Throughout this section, we assume the reader is familiar with the original UC PUF formulation of Brzuska et al. 7] (Section 4.2). 
control over the manufacturing process and may be able to produce errors in the process that break the PUF's security properties. Thus, we would like parties to rely only on the PUFs that they themselves manufacture (or obtain from a source that they trust), and not on the ones they receive from other (possibly adversarial) parties.

Malicious PUFs families. In the real world, an adversary may create a malicious PUF in a number of ways. For example, it can tamper with the manufacturing process for an honestly-generated PUF to compromise its security properties (unpredictability, for instance). It may also introduce additional behaviour into the PUF token, like logging of queries. Taking inspiration from the literature on modeling tamper-proof hardware tokens, one might be tempted to model malicious PUFs analogously in the following way: to create a malicious PUF, the adversary simply specifies to the ideal functionality, the (malicious) code it wants to be executed instead of an honest PUF. Allowing the adversary to specify the malicious code enables the simulator to "rewind" the malicious PUF, which is used crucially in security proofs in the hardware token model. However, modeling malicious PUFs in this way would disallow the adversary from modifying honest PUFs (or more precisely, the honest PUF manufacturing process). To keep our treatment as general as possible, we do not place any restriction on a malicious PUF, except that it should have the same syntax as that of an honest PUF family, as specified in Definition [1 In particular, the adversary is not required to know the code of malicious PUFs it creates, and thus our simulator can not rely on rewinding in the security proofs. Formally, we allow the adversary to specify a "malicious PUF family", that the ideal functionality uses. Of course, in the protocol, we also want the honest parties to be able to obtain and send honestly generated PUFs. Thus our ideal functionality for PUFs, $\mathcal{F}_{\text {PUF }}$ (Fig. 1) is parameterized by two PUF families: the normal (or honest) family (Sample normal, Eval $_{\text {normal }}$ ) and the possibly malicious family (Sample $e_{\text {mal }}, E$ al $_{\text {mal }}$ ). When a party $P_{i}$ wants to initialize a PUF, it sends a initpuF message to $\mathcal{F}_{\text {PUF }}$ in which it specifies the mode $\in\{$ normal, mal $\}$, and the ideal functionality uses the corresponding family for initializing the PUF. For each initialized PUF, the ideal functionality $\mathcal{F}_{\text {PUF }}$ also stores a tag representing the family (i.e., mal or normal) from which it was initialized. Thus, when the PUF needs to be evaluated, $\mathcal{F}_{\text {PUF }}$ runs the evaluation algorithm corresponding to the tag.

As in the original formulation of Brzuska et al., the ideal functionality $\mathcal{F}_{\text {PUF }}$ keeps a list $\mathcal{L}$ of tuples (sid, id, mode, $\hat{P}, \tau$ ). Here, sid is the session identifier of the protocol and id is the PUF identifier output by the Sample $e_{\text {mode }}$ algorithm. As discussed above mode $\in\{$ normal, mal $\}$ indicates the mode of the PUF, and $\hat{P}$ identifies the party that currently holds the PUF. The final argument $\tau$ specifies transition of PUFs: $\tau=$ notrans indicates the PUF is not in transition, while $\tau=$ $\operatorname{trans}\left(P_{j}\right)$ indicates that the PUF is in transition to party $P_{j}$. Only the adversary may query the PUF during the transition period. Thus, when a party $P_{i}$ hands over a PUF to party $P_{j}$, the corresponding $\tau$ value for that PUF is changed from notrans to trans $\left(P_{j}\right)$, and the adversary is allowed to send evaluation queries to this PUF. When the adversary is done with querying the PUF, it sends a readyPuF 
message to the ideal functionality, which hands over the PUF to $P_{j}$ and changes the PUFs transit flag back to notrans. The party $P_{j}$ may now query the PUF. The ideal functionality now waits for a receivedPuF message from the adversary, at which point it sends a receivedpuF message to $P_{i}$ informing it that the hand over is complete. The ideal functionality is described formally in Fig. 1,

Allowing adversary to create PUFs. We deviate from the original formulation of

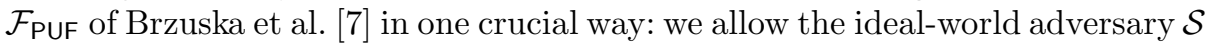
to create new PUFs. That is, $\mathcal{S}$ can send a initpuF message to $\mathcal{F}_{\text {PUF. }}$ In the original formulation of Brzuska et al., $\mathcal{S}$ could not create its own PUFs, and this has serious implications for the composition theorem. We thank Margarita Vald [35] for pointing out this issue. We elaborate on this in Appendix $\mathrm{H}$ in the full version [26]. Also, it should be noted that the PUF set-up is non-programmable, but not global [8]. The environment must go via the adversary to query PUFs, and may only query PUFs in transit or held by the adversary at that time.

We remark that the OT protocol of [7] for honest PUFs, fails in the presence of malicious PUFs. Consider the OT protocol in Fig. 3 in [7. The security crucially relies on the fact that the receiver $P_{j}$ can not query the PUF after receiving sender's first message, i.e., the pair $\left(x_{0}, x_{1}\right)$. If it could do so, then it would query the PUF on both $x_{0} \oplus v$ and $x_{1} \oplus v$ and learn both $s_{0}$ and $s_{1}$. In the malicious PUF model however, as there is no guarantee that the receiver can not learn query/answer pairs when a malicious PUF that he created is not in its hands, the protocol no longer remains secure.

PUFs and computational assumptions. The protocol we present in the next section will use computational hardness assumptions. These assumptions hold against probabilistic polynomial-time adversaries. However, PUFs use physical components and are not modeled as PPT machines, and thus, the computational assumptions must additionally be secure against PPT adversaries that have access to PUFs. We remark that this is a reasonable assumption to make, as if this is not the case, then PUFs can be used to invert one-way functions, to find collisions in CRHFs and so on, therefore not only our protocol, but any computational-complexity based protocol would be insecure. Note that PUFs are physical devices that actually exist in the real world, and thus all real-world adversaries could use them.

To formalize this, we define the notion of "admissible" PUF families. A PUF family (regardless of whether it is honest or malicious) is called admissible with respect to a hardness assumption if that assumption holds even when the adversary has access to PUFs from this family. We will prove that our protocol is secure when the $\mathcal{F}_{\text {PUF }}$ ideal functionality is instantiated with admissible PUF families. In particular, all the cryptographic tools that we use to construct our protocol can be based on the DDH assumption. From this point on in this paper, a "PUF family" would be taken to mean a PUF family which is admissible with respect to $\mathrm{DDH}$. 
$\mathcal{F}_{\text {PUF }}$ uses PUF families $\mathcal{P}_{1}=\left(\right.$ Sample $_{\text {normal }}$, Eval Eormal $)$ with parameters $\left(r g, d_{\text {noise }}, d_{\min }, m\right)$, and $\mathcal{P}_{2}=\left(\right.$ Sample $_{\text {mal }}$, Eval Eal $\left._{\text {mal }}\right)$.

It runs on input the security parameter $1^{n}$, with parties $\mathbb{P}=\left\{P_{1}, \ldots, P_{n}\right\}$ and adversary $\mathcal{S}$.

- When a party $\hat{P} \in \mathbb{P} \cup\{\mathcal{S}\}$ writes (initpuF, sid, mode, $\hat{P}$ ) on the input tape of $\mathcal{F}_{\text {PUF }}$, where mode $\in\{$ normal, mal $\}$, then $\mathcal{F}_{\text {PUF }}$ checks whether $\mathcal{L}$ already contains a tuple (sid, $*, *, *, *)$ :

- If this is the case, then turn into the waiting state.

- Else, draw id $\leftarrow$ Sample mode $\left(1^{n}\right)$ from the PUF family. Put (sid, id, mode, $\hat{P}$, notrans) in $\mathcal{L}$ and write (initializedpuF, sid) on the communication tape of $\hat{P}$.

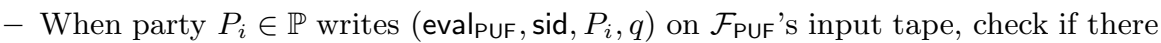
exists a tuple (sid, id, mode, $P_{i}$, notrans) in $\mathcal{L}$.

- If not, then turn into waiting state.

- Else, run $a \leftarrow \operatorname{Eval}_{\text {mode }}\left(1^{n}\right.$, id, $q$ ). Write (responsepuf, sid, $\left.q, a\right)$ on $P_{i}$ 's communication input tape.

- When a party $P_{i}$ sends (handoverpuF, sid, $P_{i}, P_{j}$ ) to $\mathcal{F}_{\mathrm{PUF}}$, check if there exists a tuple (sid, $*, *, P_{i}$, notrans) in $\mathcal{L}$.

- If not, then turn into waiting state.

- Else, modify the tuple (sid, id, mode, $P_{i}$, notrans) to the updated tuple (sid, id, mode, $\perp$, $\operatorname{trans}\left(P_{j}\right)$ ). Write (invokepuF, sid, $\left.P_{i}, P_{j}\right)$ on $\mathcal{S}$ 's communication input tape.

- When the adversary sends (eval PUF, sid, $\mathcal{S}, q$ ) to $\mathcal{F}_{\mathrm{PUF}}$, check if $\mathcal{L}$ contains a tuple (sid, id, mode, $\perp$, trans $(*)$ ) or (sid, id, mode, $\mathcal{S}$, notrans).

- If not, then turn into waiting state.

- Else, run $a \leftarrow \mathrm{Eval}_{\text {mode }}\left(1^{n}, \mathrm{id}, q\right)$ and return (responsepuF, sid, $\left.q, a\right)$ to $\mathcal{S}$.

- When $\mathcal{S}$ sends (ready $\left.\perp, \operatorname{trans}\left(P_{j}\right)\right)$.

- If not found, turn into the waiting state.

- Else, change the tuple (sid, id, mode, $\perp$, $\left.\operatorname{trans}\left(P_{j}\right)\right)$ to (sid, id, mode, $P_{j}$, notrans) and write (handoverpuF, sid, $P_{i}$ ) on $P_{j}$ 's communication input tape and store the tuple (receivedpuF, sid, $P_{i}$ ).

- When the adversary sends (received $\mathrm{PUF}$, sid, $P_{i}$ ) to $\mathcal{F}_{\mathrm{PUF}}$, check if the tuple

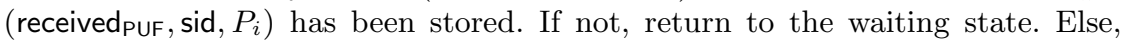
write this tuple to the input tape of $P_{i}$.

Fig. 1. The ideal functionality $\mathcal{F}_{\text {PUF }}$ for malicious PUFs

\subsection{Constructions for UC Security in the Malicious PUFs Model}

In this section we present a construction for UC-secure commitment scheme in the malicious PUFs model, which yields UC-security for any PPT functionality via the [11] compiler. 
We first recall some of the peculiarities of the PUFs model. A major difficulty when using PUFs, in contrast to say tamper-proof tokens, is that PUFs are not programmable. That is, the simulator can not simulate the answer of a PUF, and must honestly forward the queries to the $\mathcal{F}_{\text {PUF }}$ functionality. The only power of the simulator is to intercept the queries made by the adversary to honest PUFs. Thus, in designing the protocol, we shall force parties to query the PUFs with the critical private information related to the protocol, therefore allowing the simulator to extract such information in straight-line. In the malicious PUFs model the behaviour of a PUF created and sent by an adversary is entirely in the adversary's control. A malicious PUF can answer (or even abort) adaptively on the query according to some pre-shared strategy with the malicious creator. Finally, a side effect of the unpredictability of PUFs, is that the creator of a honest PUF is not able to check the authenticity of the answer generated by its own PUF, without having the PUF in its hands (or having queried the PUF previously on the very same value).

Techniques and proof intuition. Showing UC security for commitments requires obtaining straight-line extraction against a malicious sender and straight-line equivocality against a malicious receiver. Our starting point is the equivocal commitment scheme of [12] which builds upon Naor's scheme [25]. Naor's scheme consists of two messages, where the first message is a randomly chosen string $r$ that the receiver sends to the sender. The second message is the commitment of the bit $b$, computed using $r$. More precisely, to commit to bit $b$, the second message is $G(s) \oplus\left(r \wedge b^{|r|}\right)$, where $G()$ is a PRG, and $s$ a randomly chosen seed. The scheme has the property that if the string $r$ is crafted appropriately, then the commitment is equivocal. [12] shows how this can be achieved by adding a coin-tossing phase before the commitment. The coin tossing of [12] proceeds as follows: the receiver commits to a random string $\alpha$ (using a statistically hiding commitment scheme), the sender sends a string $\beta$, and then the receiver opens the commitment. Naor's parameter $r$ is then set as $\alpha \oplus \beta$.

Observe that if the simulator can choose $\beta$ after knowing $\alpha$, then it can control the output of the coin-tossing phase, and therefore equivocate the commitment. Thus, to achieve equivocality against a malicious receiver, the simulator must be able to extract $\alpha$ from the commitment. Similarly, when playing against a malicious sender, the simulator should be able to extract the value committed in the second message of Naor's commitment.

Therefore, to construct a UC-secure commitment, we need to design an extractable commitment scheme for both directions. The extractable commitment of $\alpha$ that we construct for the receiver, must be statistically-hiding (this is necessary to prove binding). We denote such commitment as $\operatorname{Com}_{\mathrm{sh}}=\left(\mathrm{S}_{\mathrm{sh}}, \mathrm{R}_{\mathrm{sh}}\right)$. On the other hand, the commitment sent by the sender, must be extractable and allow for equivocation. We denote such commitment as $\operatorname{Com}_{e q}=\left(S_{e q}, R_{e q}\right)$. As we shall see soon, the two schemes require different techniques as they aim to different properties. However, they both share the following structure to achieve extractability. 
The receiver creates a PUF and queries it with two randomly chosen challenges $\left(q_{0}, q_{1}\right)$, obtaining the respective answers $\left(a_{0}, a_{1}\right)$. The PUF is then sent to the sender. To commit to a bit $b$, the sender first needs to obtain the value $q_{b}$. This is done by running an OT protocol with the receiver. Then the sender queries the PUF with $q_{b}$ and commits to the response $a_{b}$. Note that the sender does not commit to the bit directly, but to the answer of the PUF. This ensures extractability. To decommit to $b$, the sender simply opens the commitment of the PUF-answer sent before. Note that the receiver can check the authenticity of the PUF-answer without having its own PUF back. The simulator can extract the bit by intercepting the queries sent to the PUF and taking the one that is close enough, in Hamming distance, to either $q_{0}$ or $q_{1}$. Due to the security of OT, the sender can not get both queries (thus confusing the simulator), neither can the receiver detect which query has been transferred. Due to the binding property of the commitment scheme used to commit $q_{b}$, a malicious sender cannot postpone querying the PUF to the decommitment phase (thus preventing the simulator to extract already in the commitment phase). Due to the unpredictability of PUFs, the sender cannot avoid to query the PUF to obtain the correct response.

This protocol achieves extractability. To additionally achieve statistically hiding and equivocality, protocol $\mathrm{Com}_{\mathrm{sh}}$ and $\mathrm{Com}_{\mathrm{eq}}$ develop on this basic structure in different ways accordingly to the different properties that they achieve. The main difference is in the commitment of the answer $a_{b}$.

In Protocol $\mathrm{Com}_{\mathrm{sh}}, \mathrm{S}_{\mathrm{sh}}$ commits to the PUF-response $a_{b}$ using a statistically hiding commitment scheme. Additionally, $\mathrm{S}_{\text {sh }}$ provides a statistical zeroknowledge argument of knowledge of the message committed. This turns out to be necessary to argue about binding (that is only computational). Finally, the OT protocol executed to exchange $q_{0}, q_{1}$ must be statistically secure for the OT receiver. The formal description of protocol $\mathrm{Com}_{\mathrm{sh}}$ is provided in Fig. 2.

In Protocol Com eq the answer $a_{b}$ is committed following Naor's commitment scheme. The input of $S_{e q}$ is the Naor's parameter decided in the coin-flipping phase, and is the vector $\bar{r}$ of strings $r_{1}, \ldots, r_{l}$ ( $a_{b}$ is a $l$-bit string, where $l$ is the range of the PUF). Earlier we said that the simulator can properly craft $\bar{r}$, so that it will be able equivocate the commitment of $a_{b}$. However, due to the structure of the extractable commitment shown above, being able to equivocate the commitment of $a_{b}$ is not enough anymore. Indeed, in the protocol above, due to the OT protocol, the simulator will be able to obtain only one of the PUF-queries among $\left(q_{0}, q_{1}\right)$, and it must choose the query $q_{b}$ already in the commitment phase (when the secret bit $b$ is not known to the simulator). Thus, even though the simulator has the power to equivocate the commitment to any string, it might not know the correct PUF-answer to open to. We solve this problem by asking the receiver to reveal both values $\left(q_{0}, q_{1}\right)$ played in the OT protocol (along with the randomness used in the OT protocol), obviously only after $S_{\text {eq }}$ has committed to the PUF-answer. Now, the simulator can: play the OT protocol with a random bit, commit to a random string (without querying the PUF), and then obtain both queries $\left(q_{0}, q_{1}\right)$. In the decommitment phase, the simulator gets the actual bit $b$. Hence, it can query the PUF with input 


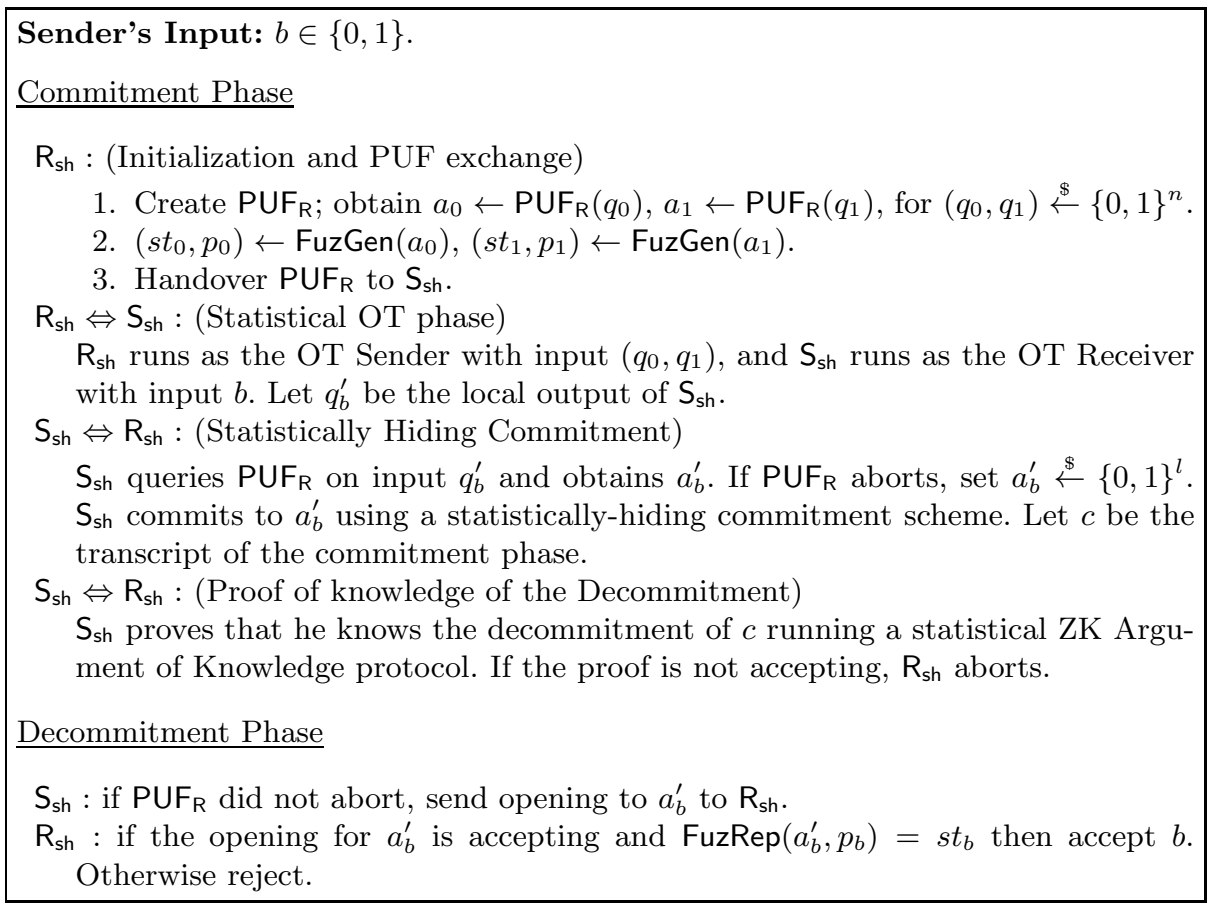

Fig. 2. Statistically Hiding Straight-Line Extractable Bit Commitment Comsh

$q_{b}$, obtain the PUF-answer, and equivocate the commitment so to open to such PUF-answer. There is a subtle issue here and is the possibility of selective abort of a malicious PUF. If the PUF aborts when queried with a particular string, then we have that the sender would abort already in the commitment phase, while the simulator aborts only in the decommitment phase. We avoid such problem by requiring that the sender continues the commitment phase by committing to a random string in case the PUF aborts. The above protocol is statistically binding (we are using Naor's commitment), straight-line extractable, and assuming that Naor's parameter was previously ad-hoc crafted, it is also straight-line equivocal. To commit to a bit we are committing to the $l$-bit PUF-answer, thus the size of Naor's parameter $\bar{r}$, is $N=(3 n) l$. Protocol Com $_{\text {eq }}$ is formally described in Fig. 3 .

The final UC-secure commitment scheme $\operatorname{Com}_{\mathrm{uc}}=\left(\mathrm{S}_{\mathrm{uc}}, \mathrm{R}_{\mathrm{uc}}\right)$ consists of the coin-flipping phase, and the (equivocal) commitment phase. In the coin flipping, the receiver commits to $\alpha$ using the statistically hiding straight-line extractable commitment scheme Com $_{\text {sh }}$. The output of the coin-flipping is the Naor's parameter $\bar{r}=\alpha \oplus \beta$ used as common input for the extractable/equivocal commitment scheme Com eq. Protocol Com $_{\mathrm{uc}}=\left(\mathrm{S}_{\mathrm{uc}}, \mathrm{R}_{\mathrm{uc}}\right)$ is formally described in Fig. 4 .

Both protocol $\mathrm{Com}_{\mathrm{sh}}$, Com $\mathrm{eq}$ require one PUF sent from the receiver to the sender. We remark that PUFs are transferred only once at the beginning of the protocol. We finally stress that we do not assume authenticated PUF delivery. 


\section{Sender's Input: Bit $b \in\{0,1\}$.}

Common Input: $\bar{r}=\left(r_{1}, \ldots, r_{l}\right)$.

\section{Commitment Phase}

$\mathrm{R}_{\text {eq }}$ : (Initialization and PUF exchange)

1. Create PUF $F_{\mathrm{R}}$. Obtain $a_{0} \leftarrow \operatorname{PUF}_{\mathrm{R}}\left(q_{0}\right), a_{1} \leftarrow \operatorname{PUF}_{\mathrm{R}}\left(q_{1}\right)$, for $\left(q_{0}, q_{1}\right) \stackrel{\$}{\leftarrow}\{0,1\}^{n}$.

2. $\left(s t_{0}, p_{0}\right) \leftarrow \operatorname{FuzGen}\left(a_{0}\right),\left(s t_{1}, p_{1}\right) \leftarrow \operatorname{FuzGen}\left(a_{1}\right)$.

3. Handover $\mathrm{PUF}_{\mathrm{R}}$ to $\mathrm{S}_{\text {eq }}$;

4. Choose random tape ranot $\stackrel{\$}{\leftarrow}\{0,1\}^{*}$.

$\mathrm{R}_{\text {eq }} \Leftrightarrow \mathrm{S}_{\text {eq }}:$ (Statistical OT phase)

$\mathrm{R}_{\text {eq }}$ runs as the OT Sender with input $\left(q_{0}, q_{1}\right)$ using randomness ranot, and $\mathrm{S}_{\text {eq }}$ runs as the OT Receiver with input $b$. Let $q_{b}^{\prime}$ be the local output of $\mathrm{S}_{\text {eq }}$. Let $\tau_{\mathrm{OT}}$ be the transcript of the execution of the OT protocol.

$\mathrm{S}_{\text {eq }}$ : (Statistically Binding Commitment)

1. $a_{b}^{\prime} \leftarrow \operatorname{PUF}_{\mathrm{R}}\left(q_{b}^{\prime}\right)$. If PUF $\mathrm{P}_{\mathrm{R}}$ aborts, $a_{b}^{\prime} \stackrel{\$}{\leftarrow}\{0,1\}^{l}$.

2. for $1 \leq i \leq l$, pick $s_{i} \stackrel{\$}{\leftarrow}\{0,1\}^{n}, c_{i}=G\left(s_{i}\right) \oplus\left(r_{i} \wedge a_{b}^{\prime}[i]\right)$. 回

3. send $c_{1}, \ldots, c_{l}$ to $\mathrm{R}_{\text {eq. }}$.

$\mathrm{R}_{\text {eq }}$ : upon receiving $c_{1}, \ldots, c_{l}$, send ranot $_{\mathrm{OT}}, q_{0}, q_{1}$ to $\mathrm{S}_{\text {eq. }}$.

$\mathrm{S}_{\text {eq }}$ : check if transcript $\tau_{\mathrm{OT}}$ is consistent with $\left(\operatorname{ran}_{\mathrm{OT}}, q_{0}, q_{1}, b\right)$. If the check fails abort.

\section{Decommitment Phase}

$\mathrm{S}_{\text {eq }}$ : if $\mathrm{PUF}_{\mathrm{R}}$ did not abort, send $\left(\left(s_{1}, \ldots, s_{l}\right), a_{b}^{\prime}\right), b$ to $\mathrm{R}_{\mathrm{sh}}$.

$\mathrm{R}_{\text {eq }}$ : if for all $i$, it holds that $\left(c_{i}=G\left(s_{i}\right) \oplus\left(r_{i} \wedge a_{b}^{\prime}[i]\right)\right) \wedge\left(\operatorname{FuzRep}\left(a_{b}^{\prime}, p_{b}\right)=s t_{b}\right)$ then accept. Else reject.

${ }^{a}$ where $\left(r_{i} \wedge a_{b}^{\prime}[i]\right)[j]=r_{i}[j] \wedge a_{b}^{\prime}[i]$.

Fig. 3. Statistically Binding Straight-line Extractable/Equivocal Commitment Comeq

Namely, the privacy of the honest party is preserved even if the adversary interferes with the delivery process of the honest PUFs (e.g., by replacing the honest PUF).

Theorem 1. If $\mathrm{Com}_{\mathrm{sh}}=\left(\mathrm{S}_{\mathrm{sh}}, \mathrm{R}_{\mathrm{sh}}\right)$ is a statistically hiding straight-line extractable commitment scheme in the malicious PUFs model, and $\mathrm{Com}_{\mathrm{eq}}=$ $\left(\mathrm{S}_{\mathrm{eq}}, \mathrm{R}_{\mathrm{eq}}\right)$ is a statistically binding straight-line extractable and equivocal commitment scheme in the malicious PUFs model, then $\operatorname{Com}_{\mathrm{uc}}=\left(\mathrm{S}_{\mathrm{uc}}, \mathrm{R}_{\mathrm{uc}}\right)$ in Fig. 4 . is a UC-secure commitment scheme in the malicious PUFs model.

The above protocol can be used to implement the multiple commitment functionality $\mathcal{F}_{\text {mcom }}$ by using independent PUFs for each commitment. Note that in our construction we can not reuse the same PUF when multiple commitments are executed concurrently $y^{4}$. The reason is that, in both sub-protocols $\operatorname{Com}_{\text {sh }}, \mathrm{Com}_{\mathrm{eq}}$,

${ }^{4}$ Note that however our protocol enjoys parallel composition and reuse of the same PUF, i.e., one can commit to a string reusing the same PUF. 
Sender's Input: Bit $b \in\{0,1\}$.

Commitment Phase

$\mathrm{R}_{\mathrm{uc}} \Leftrightarrow \mathrm{S}_{\mathrm{uc}}:$ (Coin Flipping)

1. $\mathrm{R}_{\mathrm{uc}}$ picks $\alpha \stackrel{\$}{\leftarrow}\{0,1\}^{N}$; commit to $\alpha$ by running as sender in protocol Com $_{\text {sh }}$ while $S_{\text {uc }}$ runs as receiver.

2. $\mathrm{S}_{\mathrm{uc}}$ sends $\beta \stackrel{\$}{\leftarrow}\{0,1\}^{N}$ to $\mathrm{R}_{\mathrm{uc}}$.

3. $\mathrm{R}_{\mathrm{uc}}$ sends decommitment for $\alpha$ to $\mathrm{S}_{\mathrm{uc}}$.

4. $\mathrm{S}_{\mathrm{uc}}$ : if the decommitment is not accepting, abort. Else $\mathbf{R}_{\mathrm{uc}}$ and $\mathrm{S}_{\mathrm{uc}}$ set $\bar{r}=$ $\alpha \oplus \beta$.

$\mathrm{S}_{\mathrm{uc}} \Leftrightarrow \mathrm{R}_{\mathrm{uc}}:$ (Equivocal Commitment)

$\mathrm{S}_{\mathrm{uc}}$ commit to $b$ by running as sender in $\mathrm{Com}_{\mathrm{eq}}$ where $\mathrm{R}_{\mathrm{uc}}$ runs as receiver, and the common input is $\bar{r}$.

$\underline{\text { Decommitment Phase }}$

$\mathrm{S}_{\mathrm{uc}}$ runs the decommitment phase of $\mathrm{Com}_{\mathrm{eq}}$ to open $b$.

$\mathrm{R}_{\mathrm{uc}}$ accepts if the decommitment of $b$ is accepting.

Fig. 4. Computational UC Commitment Scheme $\left(\mathrm{S}_{\mathrm{uc}}, \mathrm{R}_{\mathrm{uc}}\right)$

in the opening phase the sender forwards the answer obtained by querying the receiver's PUF. The answer of a malicious PUF can then convey information about the value committed in concurrent sessions that have not been opened yet.

When implementing $\mathcal{F}_{\text {mcom }}$ one should also deal with malleability issues. In particular, one should handle the case in which the man-in-the-middle adversary forwards honest PUFs to another party. However such attack can be ruled out by exploiting the unpredictability of honest PUFs as follows. Let $P_{i}$ be the creator of $\mathrm{PUF}_{i}$, running an execution of the protocol with $P_{j}$. Before delivering its own PUF, $P_{i}$ queries it with the identity of $P_{j}$ concatenated with a random nonce. Then, at some point during the protocol execution with $P_{j}$ it will ask $P_{j}$ to evaluate $\mathrm{PUF}_{i}$ on such nonce (and the identity). Due to the unpredictability of PUFs, and the fact that nonce is a randomly chosen value, $P_{j}$ is able to answer to such a query only if it possesses the PUF. The final step to obtain UC security for any functionality consists in using the compiler of [11, which only needs a UC secure implementation of the $\mathcal{F}_{\text {mcom }}$ functionality.

\section{Conclusion}

We introduce the Malicious PUF model which models the very realistic attack of an adversary replacing a proper PUF with a "PUF-looking" device that implements an arbitrary malicious functionality. We show that in this model is possible to achieve UC-security relying on complexity-theoretic assumptions, by providing an implementation of UC-secure commitment scheme. 
Acknowledgments. Research supported in part by NSF grants CCF-0916574; IIS-1065276; CCF-1016540; CNS-1118126; CNS-1136174; US-Israel BSF grant 2008411, OKAWA Foundation Research Award, IBM Faculty Research Award, Xerox Faculty Research Award, B. John Garrick Foundation Award, Teradata Research Award, Lockheed-Martin Corporation Research Award and the European Commission through the FP7 programme under contract 216676 ECRYPT II. This material is also based upon work supported by the Defense Advanced Research Projects Agency through the U.S. Office of Naval Research under Contract N00014-11-1-0392. The views expressed are those of the author and do not reflect the official policy or position of the Department of Defense or the U.S. Government.

The authors thank Margarita Vald for pointing out the problem with the original formulation of the 7] PUF ideal functionality where the adversary is not allowed to create PUFs. The authors also thank Marten van Dijk and Ulrich Rührmair for extensive discussions on their and our paper.

\section{References}

1. Agrawal, S., Goyal, V., Jain, A., Prabhakaran, M., Sahai, A.: New impossibility results for concurrent composition and a non-interactive completeness theorem for secure computation. In: Safavi-Naini, R. (ed.) CRYPTO 2012. LNCS, vol. 7417, pp. 443-460. Springer, Heidelberg (2012)

2. Armknecht, F., Maes, R., Sadeghi, A.-R., Standaert, F.-X., Wachsmann, C.: A formalization of the security features of physical functions. In: IEEE Symposium on Security and Privacy, pp. 397-412. IEEE Computer Society (2011)

3. Armknecht, F., Maes, R., Sadeghi, A.-R., Sunar, B., Tuyls, P.: Memory leakageresilient encryption based on physically unclonable functions. In: Matsui, M. (ed.) ASIACRYPT 2009. LNCS, vol. 5912, pp. 685-702. Springer, Heidelberg (2009)

4. Barak, B., Canetti, R., Nielsen, J.B., Pass, R.: Universally composable protocols with relaxed set-up assumptions. In: Foundations of Computer Science (FOCS 2004), pp. 394-403 (2004)

5. Barak, B., Prabhakaran, M., Sahai, A.: Concurrent non-malleable zero knowledge. In: FOCS, pp. 345-354 (2006)

6. Boit, C., Helfmeier, C., Nedospasaov, D., Seifert, J.-P.: Private Communication (2013)

7. Brzuska, C., Fischlin, M., Schröder, H., Katzenbeisser, S.: Physically uncloneable functions in the universal composition framework. In: Rogaway, P. (ed.) CRYPTO 2011. LNCS, vol. 6841, pp. 51-70. Springer, Heidelberg (2011)

8. Canetti, R., Dodis, Y., Pass, R., Walfish, S.: Universally composable security with global setup. In: Vadhan, S.P. (ed.) TCC 2007. LNCS, vol. 4392, pp. 6185. Springer, Heidelberg (2007)

9. Canetti, R., Fischlin, M.: Universally composable commitments. In: Kilian, J. (ed.) CRYPTO 2001. LNCS, vol. 2139, pp. 19-40. Springer, Heidelberg (2001)

10. Canetti, R., Kushilevitz, E., Lindell, Y.: On the limitations of universally composable two-party computation without set-up assumptions. In: Biham, E. (ed.) EUROCRYPT 2003. LNCS, vol. 2656, pp. 68-86. Springer, Heidelberg (2003) 
11. Canetti, R., Lindell, Y., Ostrovsky, R., Sahai, A.: Universally composable twoparty and multi-party secure computation. In: 34th Annual ACM Symposium on Theory of Computing, Montréal, Québec, Canada, May 19-21. LNCS, pp. 494-503. ACM Press (2002)

12. Di Crescenzo, G., Ostrovsky, R.: On concurrent zero-knowledge with pre-processing (Extended abstract). In: Wiener, M. (ed.) CRYPTO 1999. LNCS, vol. 1666, pp. 485-502. Springer, Heidelberg (1999)

13. Dodis, Y., Ostrovsky, R., Reyzin, L., Smith, A.: Fuzzy extractors: How to generate strong keys from biometrics and other noisy data. SIAM J. Comput. 38(1), 97-139 (2008)

14. Eichhorn, I., Koeberl, P., van der Leest, V.: Logically reconfigurable pufs: memorybased secure key storage. In: Proceedings of the Sixth ACM Workshop on Scalable Trusted Computing, STC 2011, pp. 59-64. ACM, New York (2011)

15. Fischlin, M., Pinkas, B., Sadeghi, A.-R., Schneider, T., Visconti, I.: Secure set intersection with untrusted hardware tokens. In: Kiayias, A. (ed.) CT-RSA 2011. LNCS, vol. 6558, pp. 1-16. Springer, Heidelberg (2011)

16. Garg, S., Kumarasubramanian, A., Ostrovsky, R., Visconti, I.: Impossibility results for static input secure computation. In: Safavi-Naini, R. (ed.) CRYPTO 2012. LNCS, vol. 7417, pp. 424-442. Springer, Heidelberg (2012)

17. Goyal, V., Ishai, Y., Sahai, A., Venkatesan, R., Wadia, A.: Founding cryptography on tamper-proof hardware tokens. In: Micciancio, D. (ed.) TCC 2010. LNCS, vol. 5978, pp. 308-326. Springer, Heidelberg (2010)

18. Guajardo, J., Kumar, S.S., Schrijen, G.-J., Tuyls, P.: Fpga intrinsic pufs and their use for ip protection. In: Paillier, P., Verbauwhede, I. (eds.) CHES 2007. LNCS, vol. 4727, pp. 63-80. Springer, Heidelberg (2007)

19. Kalai, Y.T., Lindell, Y., Prabhakaran, M.: Concurrent general composition of secure protocols in the timing model. In: 37th Annual ACM Symposium on Theory of Computing, pp. 644-653 (2005)

20. Katz, J.: Universally composable multi-party computation using tamper-proof hardware. In: Naor, M. (ed.) EUROCRYPT 2007. LNCS, vol. 4515, pp. 115-128. Springer, Heidelberg (2007)

21. Katzenbeisser, S., Koccabas, Ü., Rozic, V., Sadeghi, A.-R., Verbauwhede, I., Wachsmann, C.: Pufs: Myth, fact or busted? a security evaluation of physically unclonable functions (pufs) cast in silicon. In: Prouff and Schaumont [30], pp. 283301

22. Koccabas, Ü., Sadeghi, A.-R., Wachsmann, C., Schulz, S.: Poster: practical embedded remote attestation using physically unclonable functions. In: Chen, Y., Danezis, G., Shmatikov, V. (eds.) ACM Conference on Computer and Communications Security, pp. 797-800. ACM (2011)

23. Maes, R., Van Herrewege, A., Verbauwhede, I.: Pufky: A fully functional puf-based cryptographic key generator. In: Prouff and Schaumont [30], pp. 302-319

24. Maes, R., Verbauwhede, I.: Physically unclonable functions: A study on the state of the art and future research directions. In: Sadeghi, A.-R., Naccache, D. (eds.) Towards Hardware-Intrinsic Security, Information Security and Cryptography, pp. 3-37. Springer, Heidelberg (2010)

25. Naor, M.: Bit commitment using pseudo-randomness. In: Brassard, G. (ed.) CRYPTO 1989. LNCS, vol. 435, pp. 128-136. Springer, Heidelberg (1990)

26. Ostrovsky, R., Scafuro, A., Visconti, I., Wadia, A.: Universally composable secure computation with (malicious) physically uncloneable functions. Cryptology ePrint Archive, Report 2012/143 (2012), http://eprint.iacr.org/2012/143/ 
27. Pappu, R.S., Recht, B., Taylor, J., Gershenfeld, N.: Physical one-way functions. Science 297, 2026-2030 (2002)

28. Pappu, R.S.: Physical One-Way Functions. PhD thesis, MIT (2001)

29. Prabhakaran, M., Sahai, A.: New notions of security: achieving universal composability without trusted setup. In: 36th Annual ACM Symposium on Theory of Computing, pp. 242-251 (2004)

30. Prouff, E., Schaumont, P.: CHES 2012. LNCS, vol. 7428. Springer, Heidelberg (2012)

31. Rührmair, U.: Oblivious transfer based on physical unclonable functions. In: Acquisti, A., Smith, S.W., Sadeghi, A.-R. (eds.) TRUST 2010. LNCS, vol. 6101, pp. 430-440. Springer, Heidelberg (2010)

32. Rührmair, U., Katzenbeisser, S., Busch, H.: Strong pufs: Models, constructions and security proofs. In: Sadeghi, A., Tuyls, P. (eds.) Towards Hardware Intrinsic Security: Foundations and Practice, pp. 79-96. Springer (2010)

33. Sadeghi, A.-R., Visconti, I., Wachsmann, C.: Enhancing rfid security and privacy by physically unclonable functions. In: Sadeghi, A.-R., Naccache, D. (eds.) Towards Hardware-Intrinsic Security, Information Security and Cryptography, pp. 281-305. Springer, Heidelberg (2010)

34. Tuyls, P., Batina, L.: Rfid-tags for anti-counterfeiting. In: Pointcheval, D. (ed.) CT-RSA 2006. LNCS, vol. 3860, pp. 115-131. Springer, Heidelberg (2006)

35. Vald, M.: Private Communication (2012)

36. van Dijk, M., Rührmair, U.: Physical unclonable functions in cryptographic protocols: Security proofs and impossibility results. IACR Cryptology ePrint Archive, 2012: 228 (2012) 